\title{
TEMAS EN DEBATE, SECCIÓN DE LA REVISTA QUIMÁN -UnTER, 1987-1989
}

\author{
Tarantino Susana Ana María* \\ Universidad Nacional de Comahue, Argentina \\ Suan_tar@hotmail.com \\ Silva Nora Antonia* \\ Universidad Nacional de Comahue, Argentina \\ norasilva09@hotmail.com \\ Salerno Nancy Liliana* \\ Universidad Nacional de Comahue, Argentina \\ nancylilianasalerno@hotmail.com
}

Recibido: 29/10/2018 Aceptado: 30/12/2018

\section{Resumen}

Este artículo representa un nuevo avance sobre el contenido del discurso de la prensa escrita de un gremio docente, la Unión de Trabajadores de la Educación de Río Negro, durante el último trienio del gobierno de Raúl Alfonsín. Se intenta dilucidar la urdiembre discursiva a través de las once revistas denominadas "Quimán", editadas en forma regular y sistemática durante los años 1987-1989. El análisis se focaliza en una de las secciones titulada "Temas en debate", que aparece con mayor recurrencia en diez ejemplares. Para desentrañar la trama textual e iconográfica, en primer lugar, se identifican las problemáticas y las tensiones que se ponen de manifiesto en cada uno de los artículos. En segundo lugar, se organizan tres ejes de los cuales en esta ocasión se presenta el relacionado con la política de descentralización educativa en la provincia de Río Negro. Este tipo de prensa se constituye en una herramienta de poder político, generadora de debates y un medio de presión que viabiliza la apropiación y la defensa de derechos y distintas formas de resistencia ante el avance neoliberal que se consolidará en los noventa.

Palabras clave: Prensa escrita - Revistas sindicales y pedagógicas - Poder discursivo Descentralización educativa.

\begin{abstract}
This article represents a new advance on the content of the written press discourse of a teacher's union, the Union of Education Workers of Río Negro, during the last three years of the government of Raúl Alfonsín. An attempt is made to elucidate the discursive warping through the eleven journals called "Quimán", published regularly and systematically during the years 19871989. The analysis focuses on one of the sections entitled "Topics in debate", which appears with
\end{abstract}

\footnotetext{
* Especialista en Didáctica de las Ciencias Sociales con mención en Educación Ciudadana. Especialista en Jardín Maternal. Profesora en Ciencias de la Educación. Profesora en el Nivel Inicial. Integrante del equipo de investigación del Departamento de Historia de la Educación. Proyecto de investigación Tipo I: C-125. Ord. 0905/17. Período: 20172020, dirigido por la Dra. Glenda Miralles y co-dirigido por la Profesora Rosana Cipressi, de la Facultad de Ciencias de la Educación, Universidad Nacional del Comahue.

* Especialista en Didáctica de las Ciencias Sociales con mención en Educación Ciudadana. Profesora en Ciencias de la Educación. Profesora en Enseñanza Primaria. Integrante del equipo de investigación del Departamento de Historia de la Educación. Proyecto de investigación Tipo I: C-125. Ord. 0905/17. Período: 2017-2020, dirigido por la Dra. Glenda Miralles y co-dirigido por la Profesora Rosana Cipressi, de la Facultad de Ciencias de la Educación, Universidad Nacional del Comahue.

* Doctoranda en Educación. Especialista en Cultura Letrada. Especialista en Políticas Económicas. Profesora en Historia. Asistente de Docencia Regular. Docente investigadora -Categoría V-. Integrante del equipo de investigación del Departamento de Historia de la Educación. Proyecto de investigación Tipo I: C-125. Ord. 0905/17. Período: 20172020, dirigido por la Dra. Glenda Miralles y co-dirigido por la Profesora Rosana Cipressi, de la Facultad de Ciencias de la Educación, Universidad Nacional del Comahue.
} 
Revista de la Escuela de Ciencias de la Educación, año 15, nRo. 14, vol. 2, JULIO a diciembre de 2019. PÁGINAS 108-116. ISSN 18516297 (DESDE DICIEMBRE DE 2006 A DICIEMBRE DE 2017). ISSN 2362-3349 (EN LÍNEA). TEMAS EN DEBATE, SECCIÓN DE LA REVISTA QUIMÁN UNTER, 1987-1989. Tarantino Susana Ana María. Silva Nora Antonia. Salerno Nancy Liliana.

greater recurrence in ten copies. To unravel the textual and iconographic plot, in the first place, the problems and tensions that are revealed in each of the articles are identified. In the second place, three axes are organized, of which on this occasion the one related to the educational decentralization policy in the province of Río Negro is presented. This type of press is a tool of political power, generating debates and a means of pressure that enables the appropriation and defense of rights and different forms of resistance to the neoliberal advance that will be consolidated in the nineties.

Keywords: Written Press - Trade union and pedagogical journals - Discursive power Educational decentralization.

\section{Introducción}

El campo disciplinar en el que se desarrolla el presente trabajo corresponde a la Historia de la Educación Regional. Está enmarcado en un proyecto de investigación denominado "La educación en la Prensa escrita regional. Un análisis de la historia de la educación rionegrina (1973-2000) dirigido por la Dra. Glenda Miralles y co-dirigido por la Profesora Rosana Cipressi, de la Facultad de Ciencias de la Educación, UNCo (1).

Este estudio constituye una aproximación al contenido del discurso de la prensa gremial escrita en relación con el campo educativo, específicamente de un gremio docente provincial, la Unión de Trabajadores de la Educación de Río Negro (UnTER) (2) durante el último trienio de la gestión presidencial de Raúl Alfonsín (1987-1989).

Desde el advenimiento de la democracia, la vida sindical provincial se caracterizó por la reorganización y articulación gremial convirtiéndose la prensa escrita en el medio de comunicación que sentó las bases para la construcción del poder político-sindical.

Se considera importante estudiar la prensa gremial escrita por su potencialidad analítica, dado que permite entre otros propósitos, realizar el análisis del devenir de un gremio docente al interior de una sociedad determinada, la rionegrina, en un contexto temporal y espacial; como también resulta interesante conocer y recoger su cosmovisión, las representaciones sociales y valores que existen, y que la prensa quiere resaltar, motivar, construir y/o promover desde el sindicato. Es así como, la prensa gremial rionegrina al estar inserta en un proyecto nacional, también recoge o discute, postulados y/o principios de las políticas nacionales.

En esta oportunidad, se intenta incursionar en el análisis discursivo a través de un corpus documental constituido por once revistas sindicales-pedagógicas denominadas "Quimán", palabra de origen mapuche que significa "quiero saber" y la editorial enfatizaba en cada ejemplar "para transformar la educación". La misma fue editada desde el $1^{\circ}$ de mayo de 1987 en forma regular y sistemática cada tres meses aproximadamente y luego, durante 1989 su publicación fue irregular editándose sólo dos ejemplares.

Publicaciones cobraron un fuerte protagonismo político y social como formadoras de opinión y representaciones sociales entre un público lector restringido, los docentes rionegrinos. Sin embargo, a partir del análisis de la sección "Cartas de lectores" se desprende la trascendencia provincial y nacional de estos artefactos culturales (3), parafraseando a Martha Ruffini (2017), estas producciones obran y operan en y sobre las prácticas culturales de quienes las producen posibilitando a la vez ser repensadas y transformadas.

Desde una perspectiva metodológica cualitativa, se retomarán los aportes del Análisis Crítico del Discurso con sus diversas vertientes de análisis: crítico, ideológico (Van Dijk, 1999), político, institucional (Laclau y Mouffe, 1987) y análisis de las condiciones de producción y reconocimiento (Verón, 1993). Considerando la interpretación de Myriam Southwell (2008) se entiende por discurso a una constelación de significados caracterizada por ser abierta, incompleta y que involucra el carácter relacional de secuencias y la posibilidad de construir nuevos significados. De este modo se hace referencia no sólo a aquello que es dicho y escrito, sino a un conjunto de ideas y prácticas que articuladamente son capaces de producir y organizar un determinado sentido, una forma de entender y de intervenir en cualquier acto social.

Más precisamente, se abordará el estudio de una de las secciones que aparece con mayor recurrencia titulada "Temas en debate". Nuestra intención es entonces, dilucidar las tensiones que se ponen de manifiesto en cada uno de los artículos de esta sección. La misma aparece en diez de las once revistas analizadas correspondiendo su autoría a Cristina Galván en cinco publicaciones, -quien, por su lado, formó parte del equipo de redacción desde los inicios de esta producción cultural-, asimismo dos pertenecen a Roberto "Tony" Balmaceda, uno a Perla Sosa, otro a Aleardo Laria y dos al editor responsable UnTER sin una autoría específica.

Para desentrañar la trama discursiva textual e iconográfica de esta sección, en primer lugar, se identificarán las problemáticas que allí se enuncian, en el contexto histórico-social del sistema educativo provincial en el que están inmersas. En segundo lugar, se procederá al análisis de acuerdo con tres ejes posibles. 
Revista de la Escuela de Ciencias de la Educación, año 15, NRO. 14, vol. 2, JULIO A diciembre de 2019. PÁGINAS 108-116. ISSN 18516297 (DESDE DICIEMBRE DE 2006 A DICIEMBRE DE 2017). ISSN 2362-3349 (EN LíNEA). TEMAS EN DEBATE, SECCIÓN DE LA REVISTA QUIMÁN UNTER, 1987-1989. Tarantino Susana Ana María. Silva Nora Antonia. Salerno Nancy Liliana.

\section{Peculiaridades de un hacer}

En cuanto a la edición y publicación de la revista "Quimán", la misma dependió de la dirección de Héctor Roncallo, cuya labor y pensamientos se reflejaron en las notas editoriales junto a un equipo de redacción comprometido en la tarea asumida bajo la iniciativa de Mirtha Pagani con el incansable trabajo de Cristina Galván, Alicia Alcoleas y Virginia Ruiz. Otros se fueron incorporando paulatinamente como colaboradores, entre muchos que contribuyeron con el correr de los meses desde distintas seccionales, donde se dispusieron corresponsalías, con presentaciones esporádicas de artículos y entrevistas. Se logró el asesoramiento periodístico a través del Departamento de Ciencias de la Información y Comunicación Social, dependiente de la Facultad de Derecho y Ciencias Sociales (UNCo), con sede en la ciudad de General Roca, por un breve tiempo. Y finalmente, para la diagramación actuó Horacio Liceras, vinculado al diario Río Negro.

Estas revistas se caracterizaron por sus tapas a color con significativas fotografías en sintonía con la iconografía seleccionada en su armado, un formato de veinte por treinta centímetros y un total de páginas que osciló entre dieciocho hasta cincuenta según el número analizado.

En cada ejemplar se redactaba una nota editorial a cargo del Secretario General de la UnTER y un sumario detallado. De este modo, se diagramaron secciones recurrentes relacionadas por un lado con la organización e información gremial que abarcó temáticas tales como legislación escolar, beneficios de la obra social, jubilaciones y retiros, formación de gremialistas, salud laboral, jardines maternales. Y por otro lado, hubo secciones vinculadas a lo pedagógico: "Pueblos Originarios" (Mirtha Pagani, Tony Balmaceda); "Educación de Adultos" (Orlando Balbo, Juan C. Llorente y Silvia C. de la Plaza); "Educación Rural" (Tony Balmaceda, Fernando Russo y otros); "Derechos Humanos" (Noemí Labrune), "Temas en Debate" (Cristina de Cano Galván, Perla Sosa); "Nutrición y Aprendizaje" (Carmen Bonet); “Abajo el almidón” (Carlos Galván); "Comentando libros" (Virginia Ruiz) y "Carta de lectores”.

\section{Problemáticas que interpelan}

A partir del análisis de los diez artículos que se publican en la sección "Temas en debate" se infiere que en gran parte de ellos se invita al interlocutor o posible lector a tomar posición ante un tema que se problematiza. Al ser interpelados permitiría abrir una discusión colectiva que generaría una conciencia crítica-reflexiva en función de modificar la propia praxis docente.

De esa manera, se agruparon las problemáticas en torno a tres ejes significativos:

- La política de descentralización educativa en la provincia de Río Negro.

- El rol docente y su configuración identitaria

- La práctica didáctico-pedagógica y su relación con la concepción de un nuevo sujeto pedagógico.

En esta ocasión se presentará el relacionado con la política de descentralización educativa en la provincia de Río Negro. Nos parece pertinente, aquí, contextualizar desde cuándo se inicia en nuestro país, el proceso de descentralización en el área educación.

En la Argentina la descentralización consistió en una redistribución de las funciones y responsabilidades entre el nivel central del Estado y los niveles locales. Las bases institucionales e históricas de un Estado federal condujeron a una descentralización apoyada en los estados provinciales más que a una redefinición de las relaciones entre el Estado y otros actores sociales en materia educativa.

El concepto de "descentralización" puede denotar múltiples sentidos. Los procesos efectivos de descentralización pueden ser entendidos como situaciones de negociación de sentidos por parte de distintos actores de la política educativa.

En términos generales, los sentidos de la "descentralización" pueden ser:

1. Proceso de devolución de algo que fue arrebatado por el Estado a los particulares o municipalidades. El Estado central "devuelve" a la sociedad civil o a instancias o niveles locales del estado, la soberanía sobre distintas áreas de la acción social. En este caso, la descentralización puede ser entendida como "democratización" de la gestión del sistema.

2. Proceso de delegación de funciones de un órgano central a unidades regionales más pequeñas, pero sin confiar facultades de decisión más allá de ciertos límites. Este proceso puede ser entendido como "desconcentración administrativa".

3. Transferencia de autoridad, recursos y obligaciones de la administración central a los gobiernos de los estados. El gobierno central conserva funciones normativas, y capacidad de asignación de recursos fiscales. Este caso puede ser entendido como "federalización".

En el caso argentino, una combinación de los tres sentidos se puso en juego a lo largo de la historia del proceso de descentralización. El primer intento de transferencias y descentralización del sistema educativo se desarrolló sobre fines de la década de los '60, donde a partir de la Ley No. 17.878 el Poder Ejecutivo logró transferir sólo 680 escuelas ubicadas en las Provincias de Buenos Aires, Río Negro y La Rioja.

Una década después, en 1978, la casi totalidad de establecimientos primarios bajo dependencia nacional fueron transferidos a las jurisdicciones. 
Es indudable que la preocupación más importante de la dictadura militar, con la transferencia compulsiva de servicios educativos en 1978, era alivianar al Estado nacional de la responsabilidad de sostener y administrar buena parte del sistema educativo. Según la perspectiva de los promotores del proceso de descentralización en la Argentina, la transferencia de servicios educativos producida en 1992 se fundaba, sobre todo, en el argumento de la federalización. De acuerdo con éste, la transferencia de servicios educativos a las provincias significaba la restitución de la soberanía provincial sobre la educación, establecida inicialmente para la educación de primeras letras, en la Constitución Nacional de 1853. Las políticas educativas de los noventa en la Argentina combinaron una federalización del sistema con mecanismos centralizados de control, con el objeto de garantizar la autonomía de los estados provinciales en el área educativa a la vez que los principios de la unidad nacional.

En cambio, perspectivas críticas del proceso de descentralización, señalan que la transferencia de servicios educativos era una forma de descargar al Estado nacional de la responsabilidad sobre el financiamiento de estos servicios, al tiempo que marcaron las dificultades de algunas jurisdicciones para adecuar sus estructuras administrativas y de gestión a los nuevos servicios a su cargo. Algunas otras identificaron como una contradicción el hecho de que, junto con la transferencia de los servicios educativos, el Estado nacional retuviera responsabilidades asociadas a la evaluación, la distribución de recursos, la capacitación, etc.

\section{La política de descentralización educativa en la provincia de Río Negro}

Cristina Galván en la nota "Que cara está la educación gratuita" (4) analizaba el concepto de gratuidad en el ámbito educativo apelando a bases jurídicas partiendo de la Ley Nacional de Educación $N^{\circ} 1420$ y de la constitución de la provincia de Río Negro (1957). Se interrogaba sobre la existencia y el funcionamiento de las cooperadoras escolares cuyo marco normativo obedecía a la última dictadura militar en 1977. Estas entidades cobraban importancia al asumir responsabilidades al tratar de paliar el déficit que no cubría el Estado. Esta realidad acentuaba las diferencias socio-económicas y de funcionamiento de las escuelas, como así también, los distintos niveles de participación y decisión de los padres. Se cuestionaba así, el rol del Estado que debía ser el garante de las condiciones edilicias y de equipamiento de todos los establecimientos escolares de la provincia. En consecuencia, señalaba como propuestas alternativas posibles de discusión, en base al principio de solidaridad, las siguientes:

- Creación de un fondo común de cooperadoras. Que los padres aporten, de acuerdo a sus posibilidades, pero que el ingreso se distribuya para todas las escuelas por igual.

- Este fondo común no estará destinado a obras de infraestructura ni mantenimiento de edificios, porque esta tarea le corresponde exclusivamente al Estado.

- Los fondos recaudados si podrán servir para la adquisición de material didáctico, y excursiones educativas que serán compartidas por varias escuelas (p.29).

Rescataba de la ley 1420 dos instituciones claves que servirían como nexos con la sociedad civil y el Consejo Nacional de Educación, las cooperadoras (inc. $4^{\circ}$ del Art.42) y los Consejos Escolares (Art.38). Ambas estaban mencionadas en la Constitución de la provincia de Río Negro de 1957 (Art. 158) y retomadas en la reforma constitucional de junio de 1988 (Art. 65) que también aludían a los Consejos Escolares con carácter electivo e integrados por docentes y vecinos residentes del lugar, centrando sus funciones en la administración local y el gobierno inmediato de las escuelas. A juicio de la autora deberían funcionar como vías democráticas de convivencia "posibilitando que la mayoría de las escuelas y por lo tanto, docentes y padres abandonen su posición de islas en un entorno que exige transformaciones para lograr una sociedad más justa y humana" (p. 29).

Se observa la intencionalidad de cuestionar los valores reinantes de la época que llevaban consigo vestigios autoritarios reflejados en el control, disciplinamiento e individualismo a lo que contraponía los principios de solidaridad y convivencia mancomunada.

Este artículo se acompañaba de una fotografía de dimensiones considerables que representaba un reclamo colectivo con un cartel en primer plano, que denunciaba las distintas acciones de asistencialismo, en detrimento de la labor pedagógica realizadas por los educadores frente a la difícil coyuntura de crisis socio-educativa.

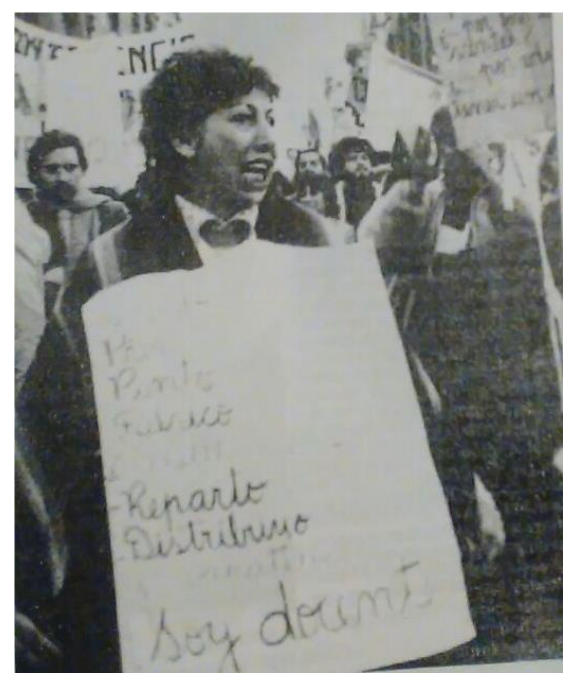


ReVISta de LA Escuela de Ciencias de la Educación, año 15, NRo. 14, vol. 2, JULIO a diciembre de 2019. PÁGINAS 108-116. ISSN 18516297 (DESDE DICIEMBRE DE 2006 A DICIEMBRE DE 2017). ISSN 2362-3349 (EN LínEA). TEMAS EN DEBATE, SECCIÓN DE LA REVISTA QUIMÁN UNTER, 1987-1989. Tarantino Susana Ana María. Silva Nora Antonia. Salerno Nancy Liliana.

Figura 1: La protesta callejera

Fuente: Quimán, 2(5), Mayo/Junio, 1988. Pág.29.

Archivo personal del Secretario General de UnTER Profesor Héctor Roncallo

El artículo titulado "La UnTER y los consejos escolares locales" (5) fue redactado y publicado sin especificar una autoría determinada, por ello, se infiere que el protagonismo recae en la dirección del profesor Héctor Roncallo, y por ende, en la Secretaría de Prensa de la UnTER. La intencionalidad que se perseguía era la búsqueda de un exhaustivo debate sindical en relación a la organización e implementación de los Consejos Escolares locales en el marco de la discusión en la Legislatura de un anteproyecto de ley de Educación Provincial tendiendo a superar la primera Ley de Educación N²27 existente desde 1961.

Por esto, presentaban en la sección "Temas en Debate" una propuesta concreta para la constitución de los Consejos Escolares Locales, su régimen de elecciones, inhabilidades, incompatibilidades y excusaciones de su funcionamiento a través de la planta funcional, las sesiones, atribuciones y funciones, entre otros considerandos.

En su análisis resulta recurrente, el siguiente enunciado: "los Consejos Escolares tendrán a su cargo la administración local y el gobierno inmediato de las escuelas de su radio, en cuanto no afecten a la PARTE TÉCNICA" (p.18). De este modo, el gremio en forma enfática niega la posibilidad de que estos consejos tuvieran incumbencia en lo "técnico". Se advierte que dicha categoría fue utilizada originalmente en la Constitución Provincial de 1957, Art. 158, presumimos que se adecuó a una política de Estado de carácter desarrollista, de allí su recurrencia. Sin embargo, se replicó en la reforma constitucional de 1988 (Art. 65) (6).

Por otra parte, se pueden destacar los mecanismos democráticos sugeridos en la elección de delegados con la organización de asambleas en cada comunidad educativa, el empoderamiento de los padres, alumnos, el gremio y sobre todo en el nivel medio, superior y de adultos incentivaba la formación de los Centros de Estudiantes para su representatividad y participación de los mismos. Se observa la incorporación de alumnos y padres como sujetos activos e incluso se refiere a la elección de ciudadanos en calidad de consejeros en lugar de vecinos.

Se contempla la creación de un cuerpo médico auditor para la atención de las necesidades de los educandos. Esta acción intenta dar respuestas a situaciones de marginalidad y pobreza extrema de la población infanto-juvenil complementando así la tarea del Servicio de Apoyo Técnico (SAT) creado con un nuevo carácter interdisciplinario según la resolución №1831/1986 y cuyos antecedentes se remontan a la época dictatorial como equipos técnicos docentes (Res. 123/78) (7). Asimismo, al analizar las atribuciones y funciones anexas de los Consejos Escolares surge la duda, si las comunidades educativas en general, estaban en condiciones humanas, materiales, intelectivas y si contaban con la predisposición para tal protagonismo y el grado de compromiso civil exigido. A continuación, se detallan algunas de sus funciones:

... Administrarán los fondos que por delegación le asignen. Gestionarán la provisión de muebles, útiles, material didáctico y demás elementos necesarios para la labor educativa. Serán los organismos de distribución... Solicitarán o implementarán los medios para el logro de las asignaciones familiares para los hogares con niños en edad escolar que se hallaren en precarias condiciones económicas. Realizarán el Censo de BIENES DEL ESTADO. Podrán efectuar adquisiciones, contrataciones y pagos, siempre y cuando los montos hayan sido previstos en las asignaciones presupuestarias... la elaboración final del PRESUPUESTO que se pondrá a consideración del Poder Ejecutivo por parte del Consejo Provincial de Educación... (p.20).

Podrán realizar refacciones o ampliaciones de edificios, previo concurso de precios. Serán los supervisores inmediatos de las obras.... Administrará el plan de Comedores Escolares de la localidad... Los recursos de los Consejos Escolares serán los que se les asigne anualmente en el presupuesto provincial. Las partidas que se le asignen anualmente en los presupuestos municipales... pagarán al personal docente que cubre suplencias de corta duración. Pagarán además la asignación por MOVILIDAD.... De los puntajes del personal docente... podrán hacer reclamos, pero NO Designar, Mover o Sacar Personal (p. 21).

En este artículo aparece una fotografía que muestra la participación de un grupo de personas menores en un aula conformando una posible asamblea que daría lugar a un debate. 


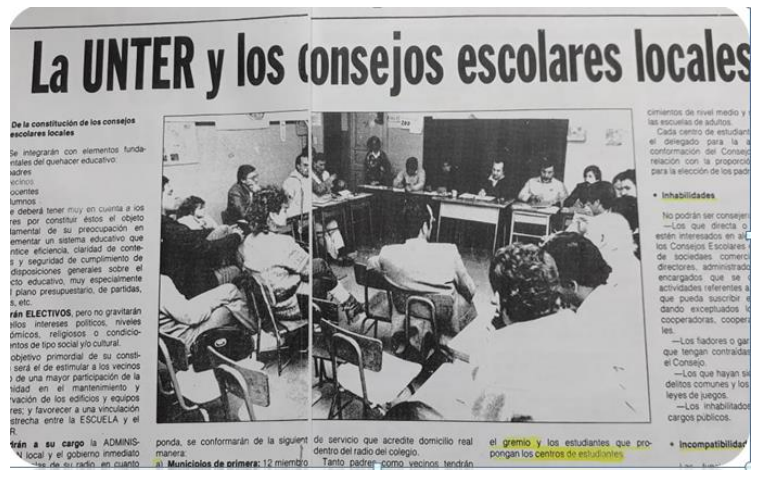

Figura № 2: Participantes en asamblea

Fuente: Quimán 3(9), Marzo/Abril 1989, pág. 18 y 19 Archivo personal del Secretario General de UnTER Profesor Héctor Roncallo

Aleardo Laría, maestro y periodista del Diario Río Negro, en un artículo que se reproduce en Quimán denominado “¿Cuántos niños no van a la escuela en la provincia de Rio Negro?” (8) hace referencia, por un lado, a la problemática de la deserción escolar, el analfabetismo y la falta de estadísticas reales tanto en el ámbito nacional como en el provincial, sobre los niños/as y jóvenes que no asistían a la escuela.

Cabe señalar que el problema del analfabetismo ya estaba contemplado en el art 163 de la constitución provincial de 1957, donde se mencionaba la importancia de la superación de tal flagelo y por tanto debieran organizarse acciones directas para tal fin. Por otro lado tal planteo servía como aporte para la discusión del anteproyecto de la nueva ley orgánica de Educación de Río Negro, poniendo al descubierto la manipulación y omisión de datos estadísticos por parte de funcionarios del área educativa; de este modo resaltaba:

... escogemos una parte en la que un funcionario (presidente del CPE) se refiere con entusiasmo a las cifras crecientes de la inscripción de alumnos entre los años 1983 y 1989; pero sin hacer simultáneamente mención alguna respecto de la deserción infantil y el analfabetismo de adultos en la cohorte 1982/1988 (p.10).
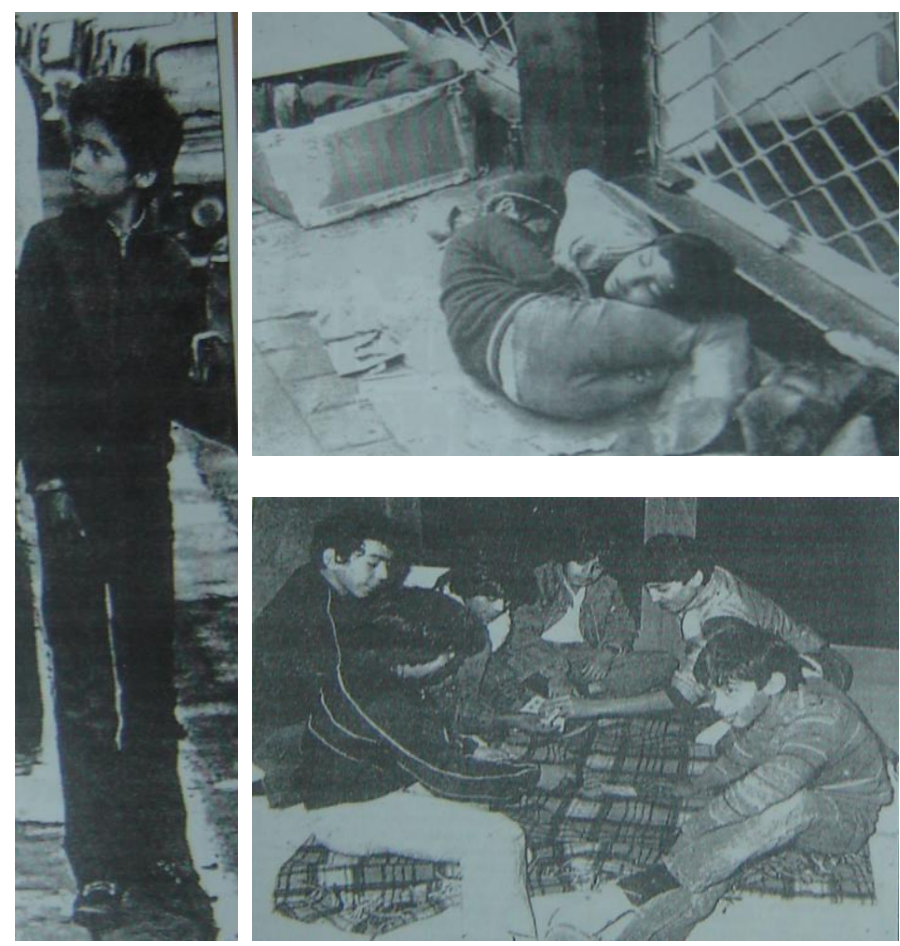
ReVISta de LA Escuela de Ciencias de la Educación, año 15, NRo. 14, vol. 2, JULIO a diciembre de 2019. PÁGINAS 108-116. ISSN 18516297 (DESDE DICIEMBRE DE 2006 A DICIEMBRE DE 2017). ISSN 2362-3349 (EN LínEA). TEMAS EN DEBATE, SECCIÓN DE LA REVISTA QUIMÁN UNTER, 1987-1989. Tarantino Susana Ana María. Silva Nora Antonia. Salerno Nancy Liliana.

\section{Figura №3: La infancia en situación de marginalidad}

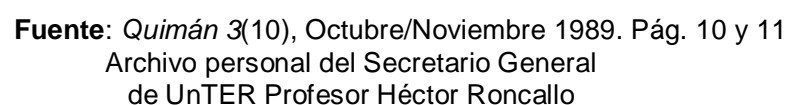

Esta nota se ilustra con tres fotografías que muestran el desamparo de niños viviendo a la intemperie en una situación de marginalidad para el paradigma de la época -vulnerabilidad en la concepción actual. De este modo, quedaba en evidencia el abandono del Estado en un contexto económico-social de inestabilidad y de creciente conflictividad social.

El autor cierra su nota manifestando la necesidad de formar Consejos Escolares por municipio, de acuerdo al anteproyecto de ley citado para concretar los censos anuales en relación a la población escolar que serviría para realizar un seguimiento pormenorizado. Además, proponía acciones tales como una ficha y una cédula escolar que afianzaría el compromiso de los padres para con sus hijos en el proceso de obligatoriedad de la enseñanza, tal como lo establece la Ley Nacional de Educación $\mathrm{N}^{\circ}$ 1420.

En el artículo, "La salud de los trabajadores de la educación" (9) Balmaceda planteaba la identidad de los docentes como trabajadores que defienden sus derechos a tener un salario digno y condiciones de vida y trabajo que garanticen el bienestar y la salud del colectivo. La imagen que acompaña el artículo periodístico es una caricatura que refleja apelando al humor la crítica situación de la docencia.

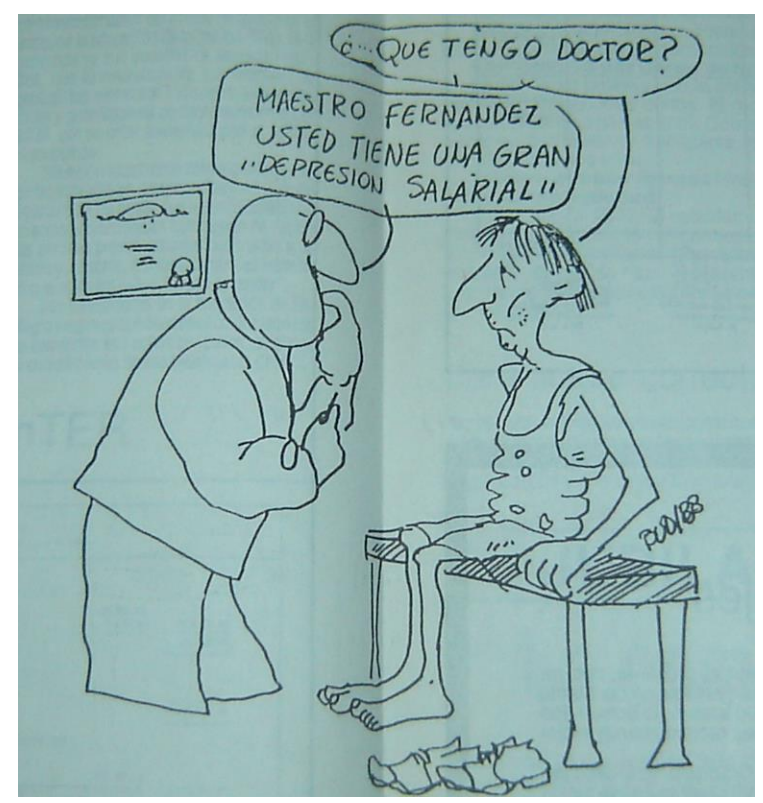

Figura $N^{\circ}$ 4: La salud docente en estado crítico

Fuente: Quimán, 2(8), Octubre/Noviembre 1988, pág. 18 y 19. Archivo personal del Secretario General de UnTER Profesor Héctor Roncallo

Cuestionaba la intención del gobierno de cercenar el régimen de licencias de la provincia de Río Negro, -Decreto 317-, (10) logro del sindicato en los años setenta, cuando el mismo se había organizado poniendo en discusión el dispositivo de control a través de la figura del "contralor médico". Y en este sentido, indicaba:

... desde la perspectiva patronal del Estado, quizás el interrogante suscite gran escozor y recargue el peso de sus hipótesis sobre la enfermedad y no tanto sobre la salud (bienestar), tornando deseables, medidas de mayor control social. E intente actualizar viejas aspiraciones de cercenar el derecho de licencias (mutilación de la 317) o implementar mecanismos de sanción encubiertas que ... recorte lentamente el derecho o rigidice y tense las relaciones interpersonales ... querrán apelar a la intensificación del control, por medio del contralor médico, recurriendo a la tradicional y siempre a mano figura del médico (p. 18).

Señalaba de este modo, las diferencias entre un sector público en constante deterioro y un sector privado que paulatinamente se fortalecía al amparo gubernamental. Por ello, interpelaba a los lectores del siguiente modo: 
Revista de la Escuela de Ciencias de la Educación, AÑo 15, NRO. 14, Vol. 2, JULIO A diCIEMBRe de 2019. PÁGINAS 108-116. ISSN 18516297 (DESDE DICIEMBRE DE 2006 A DICIEMBRE DE 2017). ISSN 2362-3349 (EN LÍNEA). TEMAS EN DEBATE, SECCIÓN DE LA REVISTA QUIMÁN UNTER, 1987-1989. Tarantino Susana Ana María. Silva Nora Antonia. Salerno Nancy Liliana.

Todo esto exige abrir una discusión y trabajo, alrededor del tema SALUD DE LOS TRABAJADORES DE LA EDUCACIÓN...Luchando por nuestra salud educamos y nos educamos, enseñamos y aprendemos, reconstruimos a la identidad que la división del trabajo (intelectual y manual) nos quitara, pudiendo sentirnos orgullosos de ser docentes y trabajadores; trabajadores y gremialistas; gremialistas y políticos; docentes y padres en todo momento y lugar (p. 19).

\section{Reflexiones finales}

Las revistas sindicales y pedagógicas denominadas Quimán tuvieron una gran repercusión no sólo en los ámbitos propios de la docencia provincial sino también nacional y latinoamericano. Su diseño y calidad tanto de su formato como de sus artículos agrupados en secciones se constituyeron en una usina generadora de interrogantes dando lugar a debates y de ese modo, incentivar la participación y el compromiso de los docentes.

A lo largo de sus páginas, lograron poner en tensión una serie de representaciones que se tenían naturalizadas como por ejemplo la de la infancia, los pueblos originarios con el themata civilización y barbarie, la escuela, el gremio, el rol docente, entre otros.

También contribuyeron en el proceso de construcción identitaria en una doble dimensión, por un lado, la formación sindical y por otro, una formación didáctica-pedagógica frente a la necesaria transformación de una realidad social integral.

La sección analizada denominada "Temas en debate" presentó una amplitud de enfoques, perspectivas y posicionamientos que favorecían a la reflexión y toma de conciencia del colectivo docente. En relación al proceso de descentralización educativa en el ámbito provincial y nacional, se plantean discusiones sobre el avance de dicha política que se puede observar tanto en nuevas figuras, como el contralor médico -poniendo en tensión el régimen de licencias, la existencia de los Consejos Escolares Locales como así también la manipulación de las estadísticas en contraste con el paulatino y sostenido desgranamiento escolar, mal llamado deserción. Estos debates constituyeron la antesala del anteproyecto de la Ley General de Educación Provincial.

La potencialidad analítica posibilita dilucidar los posicionamientos sindicales en el campo de las políticas educativas como también las representaciones sociales, valores y perspectivas prácticas que organizan el modo de mirar y leer la dinámica de la realidad social.

De este modo, se puede afirmar que Quimán se constituyó en una herramienta de difusión, de defensa y también de presión en la arena política como podremos demostrar en posteriores avances de nuestra investigación.

Para cerrar este artículo se recuperan expresiones de Héctor Roncallo, Secretario General de la UnTER en períodos sucesivos, en la editorial de uno de los primeros números:

Para hacer entender que no queremos que se nos cercenen nuestros derechos y que sí queremos una educación digna y una jerarquización acorde, produzcamos [...] el debate, la discusión, el análisis en cada lugar de trabajo observando nuestra realidad y juntos en las respectivas asambleas peticionar que nos escuchen... (Quimán, 2(7) Septiembre/Octubre 1988, p.3).

\section{Notas Bibliográfica}

(1) Miralles y co-dirigido por la Profesora Rosana Cipressi, de la Facultad de Ciencias de la Educación, UNCo. Asimismo, esta temática ha formado parte del trabajo integrador final de la profesora Nancy L. Salerno en la Carrera de Posgrado: Especialización en Cultura Letrada en la Argentina, como también una línea de investigación que se profundizará en la carrera de posgrado correspondiente al Doctorado en Educación.

(2) Los inicios se remontan a los años cincuenta con centros de magisterios que se unieron en una "Federación de Docentes Rionegrinos" y que tuvo como campo de acción el Territorio Nacional que pasó a constituirse en provincia de Río Negro durante 1957 (merced a la ley № 14408/55, de provincialización de Territorios Nacionales, en la gestión peronista). A mediados de 1974 esta federación se transformó en "Unión de Trabajadores de la Educación de Río Negro" (UnTER) en un Congreso Extraordinario realizado en San Antonio Oeste. En esos días, 29 y 30 de junio también se debatió su ingreso a CTERA y a su vez, en la CGT. Ya en 1982, según relata Roncallo, secretario gremial, hubo un paro docente en plena dictadura e incluso se logró forjar una "Federación de padres". En el mes de septiembre comenzó un período de reorganización gremial con campañas de afiliación en distintas localidades de la provincia. Ampliar en www.unter.org.ar/node/3000

(3) Cfr. Ballesteros, P. Caxias Do Sul, Brasil, 10 -03-1988. Revista Quimán 2(5), p. 32 y Ballesteros, P. Caxias do Sul, 17-02-1989 Revista Quimán 3(9), p.34.

(4) Cfr. Revista "Quimán" 2(5), Mayo/Junio de 1988, pp.28 y 29

(5) Cfr. Revista "Quimán" 3(9), Marzo/Abril de 1989, pp. 18 y 19

(6) Cabe la aclaración que en la Ley Orgánica de Educación №24444/91 no aparece la mención de la función técnica en relación a los Consejos Escolares.

(7) Véase en http://www.unterseccionalroca.org.ar/legislacion/items/2313?page=2 consultado el 27/9/18.

(8) Cfr. Revista "Quimán" 3(10), Octubre/Noviembre de 1989, pp. 10 y 11

(9) Cfr. Revista "Quimán" 2(8), Octubre/Noviembre de 1988, pp. 18 y 19 
ReVISta de LA Escuela de Ciencias de la Educación, año 15, NRo. 14, vol. 2, JULIO a diciembre de 2019. PÁGINAS 108-116. ISSN 18516297 (DESDE DICIEMBRE DE 2006 A DICIEMBRE DE 2017). ISSN 2362-3349 (EN LÍNEA). TEMAS EN DEBATE, SECCIÓN DE LA REVISTA QUIMÁN UNTER, 1987-1989. Tarantino Susana Ana María. Silva Nora Antonia. Salerno Nancy Liliana.

(10) El Régimen de Licencias corresponde al Decreto 317/74 mientras que el Estatuto del Docente en Río Negro se determinó en 1964, Ley 391 y su reforma fue un tema presente en las asambleas del gremio desde mediados de1987 específicamente la incorporación de cargos y el escalafón docente.

\section{Referencias Bibliográficas}

- Barco, S. y Mando, M. (1999). Bases constitucionales y legales de la educación en la provincia de Río Negro. En: Vior, S.; Barco S. y otros. Estado y educación en las provincia”. Buenos Aires: Miño y Dávila.

- Laclau, E. y Mouffe, Ch. (1987). Hegemonía y Estrategia Socialista. Hacia una radicalización de la democracia. Cap. 4. México: Siglo XXI.

- Ministerio de Educación, Ciencia y Tecnología (2003). "Descentralización y estrategias en educación. Caso Argentina".

- Roncallo, H. (2013). Río Negro: legislación básica para los trabajadores y trabajadoras de la educación. $1^{\circ}$ ed. Viedma: Mutual para el Magisterio Rionegrino.

- Ruffini, M. (2017). La Patagonia mirada desde arriba. El grupo Braun-Menéndez Behety y la revista Argentina Austral, 1929-1967. $1^{\circ}$ ed. Rosario: Prohistoria Ediciones.

- Southwell, M. (2012). "Análisis político del discurso: posiciones y significaciones para la política educativa" en Tello C. (Comp.) Política y epistemología de la investigación educativa.

- UnTER-Unión de Trabajadores de la Educación Rionegrina. Consultado el 16-05-2014. En www.unter.org.ar/node/3000.

- Van Dijk, T. (1999). "Análisis Crítico del Discurso" En: Antrhopos. Barcelona. En http://www.discursos.org/oldarticles/El\%20an\%E1lisis\%20cr\%EDtico\%20del\%20discurso.pdf Recuperado el 07-08- 2014

- Verón, E. (1993). La semiosis social. Fragmentos de una teoría de la discursividad. Barcelona: Gedisa S.A.,

\section{Fuentes Primarias:}

Revistas "Quimán" 1(0), Mayo, 1987. UnTER-CGT

........1(1), Julio/Agosto, 1987. UnTER-CGT;

........1(2), Septiembre/Octubre, 1987. UnTER-CGT;

.......1(4), Noviembre/Diciembre, 1987. UnTER-CGT;

.......2(4), Marzo/Abril, 1988. UnTER-CGT;

.......2(5), Mayo/Junio, 1988. UnTER-CGT

.......2(6), Julio/Agosto, 1988. UnTER-CGT;

.......2(7), Septiembre /Octubre 1988. UnTER-CTERA-CGT;

.......2(8), Octubre/Noviembre 1988. UnTER-CTERA-CGT;

........3(9), Marzo/Abril 1989. UnTER-CTERA-CGT;

...... 3(10), Octubre/Noviembre 1989. UnTER-CTERA-CGT.

Constitución Provincial de Río Negro (1957)

Reforma Constitucional de Río Negro (1988)

Ley Nacional de Educación N 1420/1884

Ley de Educación Provincial №227/1961

\section{Entrevistas:}

Roncallo, H. (2014, abril 04). Secretario General de UnTER. Entrevista personal. FaCE, UNCo, sede: Cipolletti (Río Negro) 